\title{
Characterization of secretomes provides evidence for adipose-derived mesenchymal stromal cells subtypes
}

Natalia Kalinina", Daria Kharlampieva², Marina Loguinova², Ivan Butenko², Olga Pobeguts², Anastasia Efimenko ${ }^{1}$, Luidmila Ageeva', George Sharonov', Dmitry Ischenko², Dmitry Alekseev², Olga Grigorieva', Veronika Sysoeva', Ksenia Rubina', Vassiliy Lazarev² and Vadim Govorun ${ }^{2}$

\begin{abstract}
Introduction: This study was aimed at deciphering the secretome of adipose-derived mesenchymal stromal cells (ADSCs) cultured in standard and hypoxic conditions to reveal proteins, which may be responsible for regenerative action of these cells.

Methods: Human ADSCs were isolated from 10 healthy donors and cultured for 3-4 passages. Cells were serum deprived and cell purity was assessed using multiple cell surface markers. Conditioned media was collected and analyzed using LC-MS with a focus on characterizing secreted proteins.
\end{abstract}

Results: Purity of the ADSC assessed as CD90+/CD73+/CD105+/CD45-/CD31- cells was greater than $99 \%$ and viability was greater than $97 \%$. More than 600 secreted proteins were detected in conditioned media of ADSCs. Of these 100 proteins were common to all cultures and included key molecules involved in tissue regeneration such as collagens and collagen maturation enzymes, matrix metalloproteases, matricellular proteins, macrophage-colony stimulating factor and pigment epithelium derived factor. Common set of proteins also included molecules, which contribute to regenerative processes but were not previously associated with ADSCs. These included olfactomedin-like 3, follistatinlike 1 and prosaposin. In addition, ADSCs from the different subjects secreted proteins, which were variable between different cultures. These included proteins with neurotrophic activities, which were not previously associated with ADSCS, such as mesencephalic astrocyte-derived neurotrophic factor, meteorin and neuron derived neurotrophic factor. Hypoxia resulted in secretion of 6 proteins, the most prominent included EGF-like repeats and discoidin I-like domains 3, adrenomedullin and ribonuclease 4 of RNase A family. It also caused the disappearance of 8 proteins, including regulator of osteogenic differentiation cartilage-associated protein.

Conclusions: Human ADSCs with CD90+/CD73+/CD105+/CD45-/CD31-/PDGFRß+/NG2+/CD146+(-) immunophenotype secrete a large array of proteins, the most represented group is comprised of extracellular matrix components. Number of secreted proteins is largely unaffected by prolonged hypoxia. Variability in the secretion of several proteins from cultured ADSCs of individual subjects suggests that these cells exist as a heterogeneous population containing functionally distinct subtypes, which differ in numbers between donors.

Keywords: Adipose-derived mesenchymal stromal cells, Secretome profiling, Hypoxic response, Regeneration, Cell therapy

\footnotetext{
* Correspondence: n_i_kalinina@mail.ru

${ }^{1}$ Faculty of Medicine, Lomonosov Moscow State University, 31-5,

Lomonosovsky av, Moscow 119191, Russia

Full list of author information is available at the end of the article
} 


\section{Introduction}

Multipotent mesenchymal stromal cells, isolated from bone marrow or adipose tissue (ADSC), enhance tissue regeneration upon transplantation [1], by guiding the amplification and differentiation of resident stem cells as well as by stimulating the growth of blood vessels and nerves [2-4]. Recent studies indicated that ADSCs effects on regeneration are mostly mediated by their ability to produce a wide range of bioactive molecules (growth factors, cytokines, etc.) [5, 6] as well as extracellular vesicles [7]. Therefore, the secretome of cultured ADSCs was suggested as an alternative for cell therapy and many efforts to decipher its contents were made using various approaches (reviewed in [8]). Several candidate factors, which mediate the beneficial effects of the ADSCs secretome on tissue regeneration, were identified, including vascular endothelial growth factor (VEGF), hepatocyte growth factor (HGF), insulinlike growth factor ( IGF-1), platelet-derived growth factor (PDGF-BB), angiopoietin-like 4 protein, and brainderived neurotrophic factor (BDNF) $[9,10]$. However, the content of factors necessary for the stimulation of tissue regeneration by ADSCs remains only partially characterized. The efficiency of ADSC-based therapies varies between different donors, but the results of the experimental and clinical studies analyzing the impact of donor-specific factors, including age, sex and concomitant disorders, on the efficiency of cell therapy were controversial [11-14]. Molecular mechanisms underlying donor-dependent variations of ADSC activities remain to be elucidated as well as a set of biomarkers, which would allow predicting ADSCs regenerative activity in vivo.

Several authors including us have used hypoxia as a tool to further enhance the regenerative potential of ADSCs, because hypoxic treatment caused coordinated changes of expression of genes involved in the stimulation of regeneration [15-17]. We analyzed secretomes of ADSCs derived from ten healthy female donors of similar age cultured in standard $\left(21 \% \mathrm{O}_{2}\right)$ or hypoxic $\left(1 \% \mathrm{O}_{2}\right)$ conditions. More than 600 secreted proteins were detected in conditioned media of ADSCs, many of which may promote tissue regeneration; their number is largely unaffected by prolonged hypoxia. Despite an identical immunophenotype, growth characteristics and differentiation abilities, only 100 proteins were common to all cultures. In addition, ADSCs from the different subjects secreted proteins which were variable between different cultures, including ones responsible for tissue regeneration. Variability in the secretion of several proteins by ADSCs of individual subjects suggests that these cells exist as a heterogeneous population containing functionally distinct subtypes which differ in numbers between patients.

\section{Methods}

ADSCs culture and conditioned medium harvesting

Human ADSCs were isolated from subcutaneous adipose tissue obtained from ten female donors during abdominal surgery [1]. All donors gave their informed consent and the local ethics committee of city clinical hospital \#31 (Moscow, Russia) approved the study protocol. All donors were $<50$ years old and did not have obesity or acute inflammation (Additional file 1: Table S1). All ADSC cultures were isolated from the same fat depot. This significantly limited the size of the initial sample. Therefore, we had to culture cells up to the third or fourth passages to collect a sufficient amount of material for analysis. Cells were cultured in AdvanceSTEM Mesenchymal Stem Cell Media containing $10 \%$ AdvanceSTEM Supplement (HyClone, South Logan, Utah, USA), $1 \%$ antibiotic-antimycotic solution (HyClone) at $37{ }^{\circ} \mathrm{C}$ in $5 \% \mathrm{CO} 2$ incubator. Cells were passaged at $70 \%$ confluency using HyQTase solution (HyClone). For the experiments, ADSCs of the third or fourth passages were seeded at a density of $7 \times 10^{3}$ cells $/ \mathrm{cm}^{2}$ on uncoated culture plates (Corning, NY, USA) a day before the experiment. ADSCs were washed thoroughly five times with $\mathrm{HBSS}$ without $\mathrm{Ca}^{2+}$ and $\mathrm{Mg}^{2+}$ supplemented with x100 MEM amino acid (Gibco, Waltham, MA USA) on a rotary platform placed in a $\mathrm{CO}_{2}$ incubator to remove any residues of AdvanceSTEM Supplement. After the last wash, HBSS was replaced by serum-free AdvanceSTEM Mesenchymal Stem Cell Media supplemented with x100 MEM amino acid (Gibco). For assessment of secretome content ADSCs were cultured either under standard conditions $\left(5 \% \mathrm{CO}_{2}\right.$ and $\left.21 \% \mathrm{O}_{2}\right)$ or under hypoxia $\left(5 \% \mathrm{CO}_{2}\right.$ and $\left.1 \% \mathrm{O}_{2}\right)$ at $37{ }^{\circ} \mathrm{C}$ for $48 \mathrm{~h}$ [18]. At the end of the incubation, conditioned medium was harvested and cultured cells were subjected to an analysis of immunophenotype and viability. The volume of harvested medium was measured and $100 \mathrm{mM}$ PMSF solution and $200 \mathrm{mM}$ EDTA solution were added to 2 and $5 \mathrm{mM}$ concentration, respectively. Cell debris was removed by centrifugation for $15 \mathrm{~min}$ at $200 \mathrm{~g}$. The resulting supernatant was filtered through a $0.45 \mu \mathrm{m}$ filter (BD Falcon, San Jose, CA, USA) and snap-frozen in liquid $\mathrm{N}_{2}$. The quantity and viability of the ADCSs were assessed at the end of the experiment using a Countess cell counter (Invitrogen, Waltham, MA, USA).

\section{ADSCs immunophenotyping}

The limited size of starting material did not allow us to perform immunophenotype analysis before the initial plating of cells. To confirm that ADSCs are multipotent mesenchymal stromal cells we analyzed their immunophenotype according to published criteria [19]. For phenotypic analysis cells were stained with four different antibody combinations: (1) CD45/IgG-PE/IgG-Per CP-Cy5.5/IgG-PC5; (2) CD45/CD7-PE/IgG-PerCP-Cy5. 5; (3) CD45/CD73-PE/CD105-PerCP-Cy5.5; and (4) CD 
45/IgG-PE/CD90-PC5. For patients 7-10 CD90-PC5 was replaced with CD90-PE and IgG-PC5 was removed. Cells from patients 2-9 were additionally stained with antibodies against neuro-glial proteoglycan 2 (NG2FITC, Chemicon), platelet-derived growth factor receptor B (PDGFRB-PE, BD Pharmingen), CD31-Alexa647 (BD Pharmingen, San Jose, CA, USA), CD34-PerCP (BD Pharmingen), and CD146-PE (BD Pharmingen). After $1 \mathrm{~h}$ staining at $+4{ }^{\circ} \mathrm{C}$ cells were washed once with PBS and analyzed live within $1 \mathrm{~h}$ using a LSR Fortessa flow cytometer (BD Biosciences) and FACSDiva software (BD). PerCPCy5.5 and PC5 were analyzed with $640 \mathrm{~nm}$ red laser excitation that directly excite Cy5.5 or Cy5 dye and 710$750 \mathrm{~nm}$ emission filter.

\section{ADSCs differentiation assays}

ADSCs abilities to differentiate into osteogenic and adipogenic directions were tested in vitro using standard differentiation and analysis protocols [11] in a normoxic environment. Briefly, osteogenic differentiation was induced by plating $6 \times 10^{4}$ of ADSCs onto a 24-well plate and incubating in AdvanceSTEM Mesenchymal Stem Cell Media containing 10 \% AdvanceSTEM Supplement (HyClone), $1 \%$ antibiotic-antimycotic solution (HyClone), containing $10^{-8} \mathrm{M}$ dexamethasone, $10 \mathrm{mM} \beta$-glycerol-2-phosphate, $0.2 \mathrm{mM}$ 2-phospho-L-ascorbic acid (Invitrogen, USA) for 21 days. Differentiation efficiency was analyzed using Alizarin Red S staining for calcium accumulation. Adipogenic differentiation was induced by three cycles of consecutive incubation for six days in growth medium containing $10^{-6}$ $\mathrm{M}$ dexamethasone, $10 \mu \mathrm{M}$ insulin, $200 \mu \mathrm{M}$ indomethacin and $0.5 \mathrm{mM}$ 3-isobutyl-1-methylxanthine (Invitrogen, USA) followed by three days incubation in growth medium containing $10 \mu \mathrm{M}$ insulin. Cells accumulated intracellular lipids which were analyzed using Oil-Red-O staining.

\section{Gel-free digestion of the protein sample with DTT}

The pellet was resuspended in $50 \mu \mathrm{l} 100 \mathrm{mM}$ ammonium bicarbonate with $10 \mathrm{mM}$ DTT (BioRad, Hercules, California, USA) and $1 \mu \mathrm{l}$ protease inhibitor Mix (GE Healthcare, Pittsburgh, PA, USA), mixed in a vortex, and heated at $100{ }^{\circ} \mathrm{C}$ for five min. After cooling to room temperature, the insoluble material was removed by centrifugation at $15,000 \mathrm{~g}$ for five min. Supernatant was separated and protein concentration was determined by Bradford (Bradford Protein Assay Kit, BioRad). In the supernatant, reduced protein disulfide bonds were alkylated with $30 \mathrm{mM}$ iodoacetamide (BioRad) in $100 \mathrm{mM}$ ammonium bicarbonate at room temperature and in the dark for $30 \mathrm{~min}$ and $10 \mathrm{mM}$ DTT (BioRad) in $100 \mathrm{mM}$ ammonium bicarbonate was added iteratively. Upon alkylated trypsin (Trypsin Gold, Mass Spectrometry Grade, Promega, Madison, WI, USA) in ratio trypsin:protein equal 1:30 was added to the supernatant and incubated at $37^{\circ} \mathrm{C}$ overnight.
For inactivation of trypsin activity a stock solution of trifluoroacetic acid (Sigma, St. Louis, MO, USA) (final concentration in sample $0.5 \%$ ) was added to the digest. Small amounts of the digest for liquid chromatography/ mass spectrometry (LC-MS) analysis were desalted on ZipTip (Millipore, Billerica, MA, USA).

\section{Liquid chromatography/mass spectrometry analysis and protein identification}

Analysis was performed on a TripleTOF $5600+$ massspectrometer with a NanoSpray III ion source (ABSciex, Concord, Ontario, Canada) coupled to a NanoLC Ultra 2D+ nano-HPLC system (Eksigent, Concord, Ontario, Canada). The high-performance liquid chromatography (HPLC) system was configured in a trap-elute mode. For a sample loading buffer and buffer A, the mix of $98.9 \%$ water, $1 \%$ methanol, $0.1 \%$ formic acid (v/v) was used. Buffer B was $99.9 \%$ acetonitrile, $0.1 \%$ formic acid (v/v). The trap column was conditioned prior to use by the same solvent as the column itself $(95 \%$ of solution A $(\mathrm{H} 20+1 \% \mathrm{MeOH}+0.1 \%$ formic acid $)$ and $5 \%$ of solution $\mathrm{B}(\mathrm{AcN}+0.1 \%$ formic acid) during $25 \mathrm{~min}$ with a flow rate of $300 \mathrm{nl} / \mathrm{min}$.

Samples were loaded on a trap column Chrom XP C18 3 microm $120 \AA 350$ microm*0.5 mm (Eksigent, Dublin, CA, USA) at a flow rate of $3 \mathrm{ul} / \mathrm{min}$ over $10 \mathrm{~min}$ and eluted through the separation column 3C18-CL-120 (3 microm $120 \AA$ A) 75 microm"150 mm (Eksigent) at a flow rate of $300 \mathrm{nl} / \mathrm{min}$. The gradient was from 5 to $40 \%$ of buffer B in $120 \mathrm{~min}$. The column and the precolumn were regenerated between runs by washing with $95 \%$ of buffer B for seven min and equilibrated with $5 \%$ of buffer B for $25 \mathrm{~min}$. To ensure the absence of carryover both the column and the precolumn were thoroughly washed with a blank trap-elute gradient that included five seven-min 5-95-95-5 \%B waves followed by 25 min $5 \%$ buffer B equilibration between different samples.

Mass spectra were acquired in a positive ion mode. The information-dependent mass-spectrometer experiment included one survey MS1 scan followed by 50 dependent MS2 scans. MS1 acquisition parameters were as follows: mass range for analysis and subsequent ion selection for MS2 analysis was $300-1250 \mathrm{~m} / \mathrm{z}$, signal accumulation time was $250 \mathrm{~ms}$. Ions for MS2 analysis were selected on the basis of intensity with the threshold of $250 \mathrm{cps}$ and the charge state from 2 to 5 . MS2 acquisition parameters were as follows: resolution of quadrupole was set to UNIT (0.7 Da), measurement mass range was $200-1800 \mathrm{~m} / \mathrm{z}$, optimization of ion beam focus was to obtain maximal sensitivity, signal accumulation time was $50 \mathrm{~ms}$ for each parent ion. Collision activated dissociation was performed with nitrogen gas with collision energy ramping from 25 to $55 \mathrm{~V}$ within $50 \mathrm{~ms}$ signal 
accumulation time. Analyzed parent ions were sent to a dynamic exclusion list for $15 \mathrm{~s}$ in order to get an MS2 spectra at the chromatographic peak apex (minimum peak width throughout the gradient was about $30 \mathrm{~s}$ ). Instrument reproducibility is controlled during routine process and technical duplicates were not run. Temporal biases were not reduced.

For protein identification, .wiff data files were analyzed with ProteinPilot 4.5 revision 1656 (ABSciex) using the search algorithm Paragon 4.5.0.0 revision 1654 (ABSciex) and a standard set of identification settings to search against Uniprot Swissprot (dated 20131002) database. The following parameters were used: alkylation of cysteine iodoacetamide, trypsin digestion, TripleTOF 5600 equipment, and species: Homo sapiens, thorough search with additional statistical FDR analysis. Peptide identifications were processed with default settings by a ProteinPilot software built-in ProGroup algorithm. Our approach allows us to detect $90 \%$ of peptides with a concentration $>5$ fMol and 1,500 of the most presented proteins. The final protein identification list was obtained with the threshold reliable protein ID unused score calculated by ProteomicS Performance Evaluation Pipeline Software (PSPEP) algorithm for $1 \%$ global FDR from fit and with the requirement for more than two unique peptides for each protein.

\section{RNA isolation and transcriptome analysis}

To confirm the expression of identified proteins, we performed gene array experiments. Total cellular RNA was isolated from normoxic ADSCs using a RNeasy Kit (Qiagen, Venlo, The Netherlands, cat \# 74104) according to the manufacturer's instructions. Five hundred nanograms of total RNA was labeled and hybridized on HumanHT-12 v4 Expression BeadChip (Cat. no. BD-1030204; Illumina, San Diego, CA, USA), according to the manufacturer's recommendations (Illumina Gene Expression Profiling Assay Guide). BeadChips were scanned with the Illumina iScan Reader. Data were imported into GenomeStudio (Illumina) and analyzed using built-in modules. Signals with detection $p$ value $<0.05$ were considered as significant.

\section{Real-time PCR}

To confirm changes of protein content under hypoxic treatment, we performed real-time PCR using total RNA isolated from normoxic and hypoxic ADSCs. CDNA was synthesized using Fermentas Reverse Transcription Reagents (Fermentas, Vilnius, Lithuania) with oligo-dT and RevertAid $^{\text {mo }}$ M-MuLV Reverse Transcriptase (Fermentas) according to the manufacturer's instructions. Real-time PCR was performed using ready-to-use reaction mix, containing DNA polymerase, SYBR Green and ROX (Evrogen, Moscow, Russia) in 7500 Fast Real-time PCR system (Applied Biosystems, South Logan, Utah, USA). The following oligonucleotide primers were used for amplification:

VEGFA: forward CAACATCACCATGCAGATTATGC, reverse GCTTTCGTTTTTGCCCCTTTC; EDIL: forward AAATGGAGGTATCTGTTTGCCAG, reverse CCCCTC GGTATGCTTCACTTATT; RNASE4: forward TGCAGA GGACCCATTCATTGC, reverse TCAAGTTGCAGTAG CGATCAC; ADML: forward TGCCCAGACCCTTATTC GG, reverse AGTTGTTCATGCTCTGGCGG; CRTAP: forward GAAGCATCCTGATGACGAAATGA, reverse A GTTCTCACCGTTGTATGCCC; HSP90AB2P: forward AGTTGGACAGTGGTAAAGAGCT, reverse TCCACTA CTTCTTTGACCTGCA; GCSF: forward CCCTCCCCA TCCCATGTATTTATC, reverse ACCTATCTACCTCCC AGTCCAG; EEF1A1: forward TGTCGTCATTGGACA CGTAGA, reverse ACGCTCAGCTTTCAGTTTATCC.

Fold change of mRNA expression in hypoxic samples was calculated using the $2^{-\Delta \Delta \mathrm{Ct}}$ method, EEF1A1 was used as a reference gene.

\section{Protein electrophoresis and Western blotting}

To confirm ADSC response to hypoxia, HIF-1 alpha content was analyzed using Western blotting. Protein electrophoresis was carried out under denaturing conditions with sodium dodecyl sulfate according to Laemmli [20]. Cells lysed in buffer with $1 \%$ Triton X-100 were separated in $10 \% 1 \mathrm{~mm}$ PAAG (30 $\mu \mathrm{g}$ of protein per lane) at $120 \mathrm{~V}$ before the tracking dye release. Protein molecular weight was estimated using a pre-stained protein ladder (BioRad). Separated proteins were transferred to a PVDF membrane (Millipore) by semi-dry electroblotting [21] at $25 \mathrm{~V}$ for $45 \mathrm{~min}$ in buffer for protein transfer. The membrane with transferred protein was incubated in phosphate buffer (PBS) with $5 \%$ fat-free milk and $0.01 \%$ Tween-20 for $1 \mathrm{~h}$. The membrane was incubated with primary mouse monoclonal antibodies to HIF-1 alpha (Abcam, Cambridge, UK) overnight, followed by four washes in PBS with $0.01 \%$ Tween- 20 . Then membranes were incubated with secondary antimouse antibodies conjugated with horseradish peroxidase (R\&D) and washed with PBS with $0.01 \%$ Tween- 20 . Protein bands were visualized with BioMax roentgen film (Kodak, Rochester, NY, USA) by a chemiluminescence technique. Luminescence was initiated by luminol reaction with hydrogen peroxide (ECL, Amersham, Pittsburgh, PA, USA) catalyzed by horseradish peroxidase conjugated with secondary antibodies. Protein amounts in samples were normalized by GAPDH protein content.

\section{Enzyme-linked immunosorbent assay}

ADSC secretomes were analyzed for accumulation of granulocyte-colony stimulating factor (G-CSF) using Quantikine enzyme-linked immunosorbent assay (ELISA) 


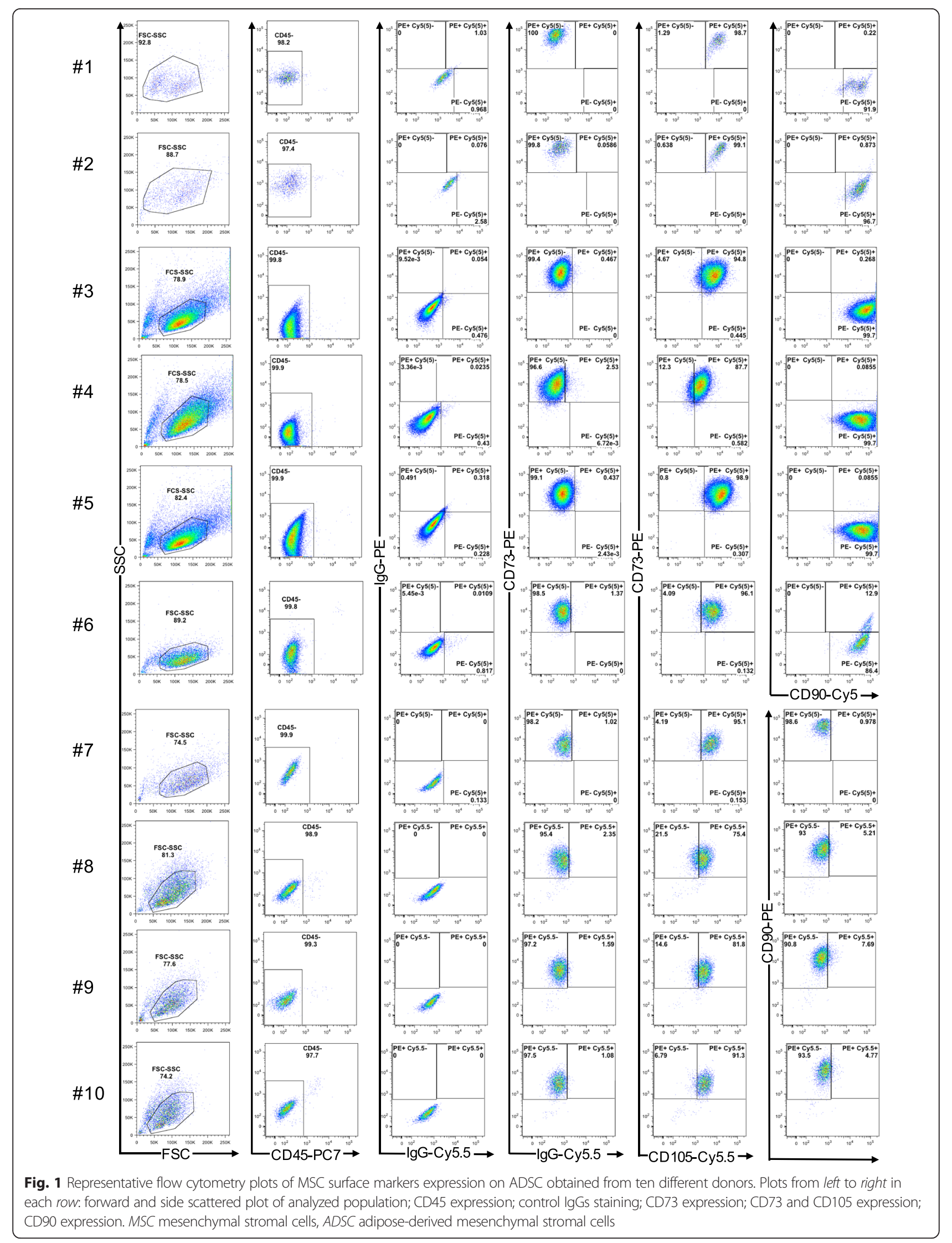


(\#DCS50, R\&D Systems, Minneapolis, MN, USA) according to the manufacturer's instructions. Concentration of G-CSF in individual samples was normalized to total protein concentration measured by Bradford assay.

\section{Statistics and bioinformatics}

Identified proteins were analyzed for the possibility of secretion using SignalP (http://www.cbs.dtu.dk/services/ SignalP), SecretomeP (http://www.cbs.dtu.dk/services/Se cretomeP) and ExoCarta (http://www.exocarta.org) databases and further subjected to bioinformatic analysis. To determine over-represented proteins for both hypoxia and control samples we used a hypergeometric test (confidence level $\mathrm{P}$-value $<=0.05$ ). Functional annotation clustering was performed using DAVID Bioinformatics Resources 6.7 (https://david.ncifcrf.gov), using default settings. Functional clusters with $p$-value $<0.05$ were considered strongly enriched in the annotation categories [22]. Statistical analysis of real-time PCR and ELISA data was performed using Statistica 8.0 software. Values are expressed as mean \pm standard error of the mean (SEM). Data were assessed for normality of distribution using the Kolmogorov-Smirnov test. Differences between treatment and control groups were then analyzed using Student's $t$ test. Statistical significance was defined as $p$-value $<0.05$.

\section{Results}

\section{Characterization of ADSCs secretomes}

ADSCs of third or fourth passages cultured in serumfree medium for $48 \mathrm{~h}$ demonstrated more than $97 \%$ viability at the end of the incubation, and $>99 \%$ of these cells possessed $\mathrm{CD} 105^{+} \mathrm{CD} 73^{+} \mathrm{CD} 90^{+} \mathrm{CD} 45^{-}$immunophenotype (Fig. 1) suggested for multipotent mesenchymal stromal cells [19]. They were capable of osteogenic and adipogenic differentiation (Additional file 2: Figure S1). More than $90 \%$ of these cells expressed the perycite markers PDGFR $\beta$ and NG2 proteoglycan. In contrast to these proteins, the expression of CD146 antigen varied between ADSCs populations from different donors, $22 \pm$ $14.5 \%$ of cells were positively stained with specific antibody (Additional file 3: Figure S2). Using LC-MS, 606 secretory proteins were identified in ADSCs conditioned medium (for protein catalogue see Additional file 1: Table S2). Of these, 452 proteins were considered as secreted through a classical pathway (endoplasmic reticulum/Golgi apparatusdependent pathway), because a signal peptide was predicted by the Signal Peptide Database, while 157 proteins were secreted through nonclassical pathways. The expression of mRNAs corresponding to the identified proteins was confirmed using Illumina Gene Expression Profiling Assay (Additional file 4: Table S3).

Despite similar anthropometric characteristics of donors included in the study, the same culture conditions, growth characteristics, immunophenotype and differentiation potential of ADSCs, only 100 proteins were common to all cultures (Additional file 1: Table S4). Functional annotation clustering has revealed 72 clusters. Those with the highest enrichment score and protein counts included extracellular matrix (ECM) proteins, proteins involved in cell adhesion, and matrix proteases as well as their inhibitors (Table 1 and Additional file 1: Table S4). Proteins common to all ADSC cultures also included molecules involved in blood vessel development, wound healing, immune response, and neuron projection development and regeneration, such as M-CSF, PEDF, DKK3, olfactomedin-like 3 , follistatin-like 1 , and prosaposin (Table 1 and Additional file 1: Tables S2 and 4).

In addition to common proteins, ADSCs from different subjects secreted proteins, which varied between different cultures (Additional file 1: Table S4). Together common and variable proteins secreted by ADSCs fit over $400 \mathrm{GO}$ terms with $p$ value $<0.05$. Interestingly, terms with the highest protein counts matched GO terms identified for common proteins. Thus, $101 \mathrm{ECM}$ proteins were identified in ADSCs secretomes (GO:0005578 proteinaceous extracellular matrix, see Additional file 1: Table S5 for proteins list), which corresponds to $30 \%$ of all known human proteins included in this category. Overall, ADSCs secretomes contained 41 proteins involved in blood vessel development (see Additional file 1: Table S6 for proteins list) and 24 proteins grouped into GO:0030182 neuron differentiation term (see Additional file 1: Table S7). These included angiopoietin-like 2 and -4 , gremlin- 1 and -2 , inhibin A, IGFBP 3-7 and -10 , IL-6 and -8 , SCF, MIF, urokinase, SDF-4, PDGF-D and VEGF-C. Several proteins, which were not previously associated with ADSCs were also found in their secretomes. These included proteins with neurotrophic activities, such as caprin 1, mesencephalic astrocyte-derived neurotrophic factor, meteorin and neuron derived neurotrophic factor.

\section{ADSCs secretome content is largely unaffected by prolonged hypoxia}

To enhance the secretion of proteins involved in tissue regeneration, we subjected ADSCs to hypoxia $\left(1 \% \mathrm{O}_{2}\right.$ for $48 \mathrm{~h}$ ) [18]. Cells responded to hypoxia in a similar manner, by $5.6 \pm 0.4$ fold up-regulation of the content of HIF-1 $\alpha$ (Additional file 5: Figure S3) as well as by $2.82 \pm$ 1.28 fold elevation of known HIF- $1 \alpha$ target VEGFA mRNA. In secretomes of hypoxic ADSCs 616 secretory proteins were identified; 480 of these were considered to be secreted through a classical pathway, while 142 proteins were secreted through nonclassical pathways (Table 2, Additional file 5: Figure S3). Overall, ADSC incubation in hypoxia resulted in secretion of six proteins which were not found in normoxic secretomes (Table 3). These included proteins involved in the regulation of angiogenesis, such as EGF-like repeats and discoidin I-like domains 3 
Table 1 Functional categories of common proteins identified in all normoxic ADSC secretomes

\begin{tabular}{|c|c|c|c|c|c|}
\hline GO term & Count $^{a}$ & $\%$ & $\begin{array}{l}P \\
\text { value**}^{* *}\end{array}$ & Proteins (Uniprot acc no.) & Examples \\
\hline $\begin{array}{l}\text { GO:0005578 proteinaceous } \\
\text { extracellular matrix }\end{array}$ & 41 & 41.4 & $<0.001$ & $\begin{array}{l}\text { P02751, P05997, P01033, P20908, P27797, } \\
\text { Q99715, P02461, Q16610, P16035, P09486, } \\
\text { P28300, P07942, P11047, P98160, P98095, } \\
\text { P12110, P14543, P12111, Q9UBX5, P02452, } \\
\text { P23142, P55268, Q16363, P35555, Q15582, } \\
\text { P08123, O95967, P07585, P24821, P51884, } \\
\text { P12109, P08254, P08253, Q08629, P21810, } \\
\text { Q12805, Q15063, Q08380, Q14767, P13611, } \\
\text { P03956 }\end{array}$ & $\begin{array}{l}\text { Collagens, collagen-maturation enzymes, } \\
\text { collagen interacting proteins, elastin- } \\
\text { associated molecules, matricellular } \\
\text { proteins, laminins }\end{array}$ \\
\hline $\begin{array}{l}\text { GO:0005509 calcium ion } \\
\text { binding }\end{array}$ & 35 & 35.4 & $<0.001$ & $\begin{array}{l}\text { Q02818, P35442, P19022, P27797, P09871, } \\
\text { Q92626, P12814, P09486, P11021, O43707, } \\
\text { P07996, P98095, P00736, P14543, Q9UBX5, } \\
\text { Q15293, P23142, Q12841, P80303, P15289, } \\
\text { P35555, O95967, O94985, Q9BRK5, P06396, } \\
\text { P13497, P08254, P08253, Q08629, P67936, } \\
\text { Q12805, O43852, Q14767, P13611, P03956 }\end{array}$ & $\begin{array}{l}\text { calsyntenin } 1 \text {; heat shock } 70 \mathrm{kDa} \text { protein } 5 \text {; } \\
\text { reticulocalbin } 1 \text {; stromal cell derived factor } \\
4 \text {; calumenin; nucleobindin } 1,2 \text {; calreticulin }\end{array}$ \\
\hline GO:0007155 cell adhesion & 31 & 31.3 & $<0.001$ & $\begin{array}{l}\text { Q16270, P35442, P02751, P20908, P19022, } \\
\text { Q99715, P02461, P12814, P07942, P11047, } \\
\text { P07996, P98160, P12110, P12111, P14543, } \\
\text { Q9UBX5, P05067, P55268, Q16363, Q15582, } \\
\text { P35579, O94985, P24821, Q8IUX7, P13497, } \\
\text { P12109, Q08629, Q15063, Q08380, O14498, } \\
\text { P13611 }\end{array}$ & $\begin{array}{l}\text { Lectin } 3 \text { binding protein; BMP1; AE binding } \\
\text { protein 1;N-cadherin; IGFBP-7; TGFBIP }\end{array}$ \\
\hline GO:0006928 cell motion & 14 & 14.1 & $<0.001$ & $\begin{array}{l}\text { P02751, P20908, P19022, P60709, P05067, } \\
\text { P35579, Q13822, P09493, P06753, Q08629, } \\
\text { P67936, P11047, P07996, P13611 }\end{array}$ & $\begin{array}{l}\text { Extracellular lysophospholipase } \\
\text { D; tropomyosins } 1,3,4\end{array}$ \\
\hline $\begin{array}{l}\text { GO:0008233 peptidase } \\
\text { activity }\end{array}$ & 13 & 13.1 & $<0.001$ & $\begin{array}{l}\text { P30101, O14773, P16870, P07858, P09871, } \\
\text { P05067, Q8IUX7, P13497, P08254, P08253, } \\
\text { P05121, P03956, P00736 }\end{array}$ & $\begin{array}{l}\text { MMP1, 2; carboxypeptidase E; cathepsin } \\
\text { B; tripeptidyl peptidase I }\end{array}$ \\
\hline $\begin{array}{l}\text { GO:0004866 endopeptidase } \\
\text { inhibitor activity }\end{array}$ & 12 & 12.1 & $<0.001$ & $\begin{array}{l}\text { P16035, P36955, P01033, P12111, P05155, } \\
\text { P05121, P11021, P50454, P05067, P07093, } \\
\text { Q6YHK3, P01034 }\end{array}$ & PEDF; TIMP1,2; PAI-1, 2; cystatin C \\
\hline $\begin{array}{l}\text { GO:0001568 blood vessel } \\
\text { development }\end{array}$ & 11 & 11.1 & $<0.001$ & $\begin{array}{l}\text { P20908, P19022, P08253, P02452, P28300, } \\
\text { P21810, Q16363, P02461, P07996, P35579, } \\
\text { P08123 }\end{array}$ & $\begin{array}{l}\text { N-cadherin; thrombospondin 1, 2; biglycan; } \\
\text { lysyl oxidase }\end{array}$ \\
\hline GO:0042060 wound healing & 10 & 10.1 & $<0.001$ & $\begin{array}{l}\text { P02751, P09493, P20908, P05155, P06396, } \\
\text { P05121, Q9UBX5, P28300, P02461, O95967 }\end{array}$ & Fibulins 4, 5; collagen, type III; fibronectin 1 \\
\hline $\begin{array}{l}\text { GO:0006955 immune } \\
\text { response }\end{array}$ & 10 & 10.1 & 0.037 & $\begin{array}{l}\text { P61769, P05155, P26022, O75326, P10909, } \\
\text { P09871, Q92626, P07996, Q13822, P00736 }\end{array}$ & $\begin{array}{l}\text { pentraxin 3; Sem7A; } \beta 2 \text {-microglobulin; } \\
\text { clusterin }\end{array}$ \\
\hline $\begin{array}{l}\text { GO:0060348 bone } \\
\text { development }\end{array}$ & 7 & 7.1 & $<0.001$ & $\begin{array}{l}\text { P05997, P13497, P08253, P09486, P02452, } \\
\text { P17936, P98160 }\end{array}$ & Perlecan; osteonectin; BMP1; IGFBP 3 \\
\hline $\begin{array}{l}\text { GO:0031175 neuron } \\
\text { projection development }\end{array}$ & 6 & 6.1 & 0.027 & $\begin{array}{l}\text { P10909, P07942, P60709, P05067, P55268, } \\
\text { P13611 }\end{array}$ & $\begin{array}{l}\text { Versican; amyloid beta (A4) precursor } \\
\text { protein }\end{array}$ \\
\hline $\begin{array}{l}\text { GO:0060284 regulation of } \\
\text { cell development }\end{array}$ & 5 & 5.1 & 0.046 & P16035, P36955, P19022, P27797, P17936 & N-cadherin; PEDF; TIMP2; IGFBP 3 \\
\hline GO:0031099 regeneration & 4 & 4 & 0.011 & P06396, P05121, P55268, P13611 & PAI-1, laminin S; gelsolin \\
\hline $\begin{array}{l}\text { GO:0007178 } \\
\text { transmembrane receptor } \\
\text { protein serine/threonine } \\
\text { kinase signaling pathway }\end{array}$ & 4 & 4 & 0.030 & Q12841, P02461, P08123, Q14767 & CD109; DKK3; follistatin-like 1 \\
\hline $\begin{array}{l}\text { GO:0016860 intramolecular } \\
\text { oxidoreductase activity }\end{array}$ & 3 & 3 & 0.027 & P30101, P07237, P41222 & $\begin{array}{l}\text { prolyl 4-hydroxylase; prostaglandin D2 } \\
\text { synthase }\end{array}$ \\
\hline
\end{tabular}

ADSC adipose-derived mesenchymal stromal cells, GO Gene Ontogeny, TGFBIP transforming growth factor, beta-induced, $68 \mathrm{kDa}$, IGFBP insulin-like growth factor binding protein

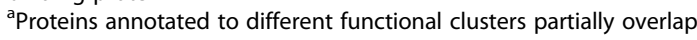

${ }^{* *} p$ value according to kappa statistics (DAVID Bioinformatics Resources, https://david.ncifcrf.gov) [22, 47] 
Table 2 ADSC secretomes overview

\begin{tabular}{|c|c|c|}
\hline Parameter & Normoxia & Hypoxia \\
\hline Total number of proteins found in secretomes & 608 & 620 \\
\hline $\begin{array}{l}\text { Number of common proteins shared by all } \\
\text { secretomes }\end{array}$ & 100 & 79 \\
\hline Number of classically secreted proteins & 451 & 480 \\
\hline $\begin{array}{l}\text { Number of proteins secreted via nonclassical } \\
\text { pathways }\end{array}$ & 157 & 140 \\
\hline Number of overrepresented proteins* & 7 & 2 \\
\hline Number of unique proteins ${ }^{a}$ & 1 & 4 \\
\hline
\end{tabular}

ADSC adipose-derived mesenchymal stromal cells

a proteins found only in normoxic or hypoxic secretomes ( $>3$ samples, $p>0.1$ ) ${ }^{*} p<0.1$ (hypergeometric test)

(EDIL), ribonuclease 4 of RNase A family and adrenomedullin. Eight proteins were found in hypoxic samples less frequently compared to normoxia (Table 3). Using realtime PCR we confirmed that hypoxia has affected the expression of corresponding mRNAs (Fig. 2). We also examined if hypoxia affects the expression of rarely detected proteins such as G-CSF, which was found only in one hypoxic but not in normoxic secretomes. Concentration of G-CSF in secretomes of normoxic ADSC was 13.4 \pm 8.5 fmol, which is close to the detection limit of LS/MS analysis. In hypoxia, G-CSF mRNA and protein were upregulated $1.7 \pm 0.3$ and $1.9 \pm 0.5$ fold, respectively (Fig. 3).

Protein distribution by functional clusters in hypoxic secretomes was similar to normoxia and the rank order or significance of functional clusters did change between normoxic and hypoxic conditions. The most represented group was GO:0005578 proteinaceous extracellular matrix (98 proteins). Within this cluster four additional proteins were detected, which have not been found in normoxic secretomes. However, these were found only in one or two secretomes each (of different donors); therefore, this finding must be considered with caution. At the same time, seven ECM proteins, which have been detected in normoxic secretomes were not found in hypoxic samples (Additional file 1: Table S5). The disappearence of only one of these, namely cartilage associated protein (CRTAP) was significant (Table 3); other proteins were found only in one or two normoxic secretomes each.

\section{Discussion}

Since ADSCs are promising material for cell therapy and tissue engineering, their secretome has been analyzed in many studies using various qualitative and quantitative approaches (for details see $[8,23]$ and references therein) in order to understand the physiology of these cells. Our data are consistent with published observations regarding ADSCs secretome content and provide an expansion of known catalogues of proteins secreted by ADSCs. Thus, $75 \%$ of identified secretory proteins possess a signal peptide and, therefore, are likely to be secreted by classical ER-Golgi apparatus pathway [24]. Other proteins are suggested to undergo unconventional secretion, via non-vesicular and vesicular pathways [25]. For example, annexin A2, which was found in the most secretomes analyzed in the current study, is produced via direct translocation across the plasma membrane [26]. ADSCs secretomes also contain proteins secreted via exosomes (e.g., inhibin A and SCF [7]) and through so called "Golgi bypass" (eg., serglycin [27]).

Table 3 Overrepresented proteins in secretomes of normoxic or hypoxic ADSCs

\begin{tabular}{|c|c|c|}
\hline Uniprot_acc \# & Protein name & $P$ value \\
\hline \multicolumn{3}{|c|}{ Proteins overrepresented in secretomes of normoxic ADSCs } \\
\hline P35556 & fibrillin 2 & 0.08 \\
\hline P35052 & glypican 1 & 0.07 \\
\hline P29401 & transketolase & 0.08 \\
\hline 075718 & cartilage associated protein & 0.04 \\
\hline P48723 & heat shock protein 70 kDa family, member 13 & 0.08 \\
\hline Q14257 & reticulocalbin 2, EF-hand calcium binding domain & 0.08 \\
\hline P30048 & peroxiredoxin 3 & 0.08 \\
\hline Q9UL46 & proteasome activator subunit 2 (PA28 beta) & 0.07 \\
\hline \multicolumn{3}{|c|}{ Proteins overrepresented in secretomes of hypoxic ADSCs } \\
\hline Q8N6G6 & ADAMTS-like 1 & 0.04 \\
\hline P19823 & inter-alpha (globulin) inhibitor $\mathrm{H} 2$ & 0.04 \\
\hline $\mathrm{O} 43854$ & EGF-like repeats and discoidin I-like domains 3 & 0.1 \\
\hline P34096 & ribonuclease, RNase A family, 4 & 0.1 \\
\hline P35318 & adrenomedullin & 0.1 \\
\hline P78324 & Tyrosine-protein phosphatase non-receptor type substrate 1 (SIPRA) & 0.1 \\
\hline
\end{tabular}



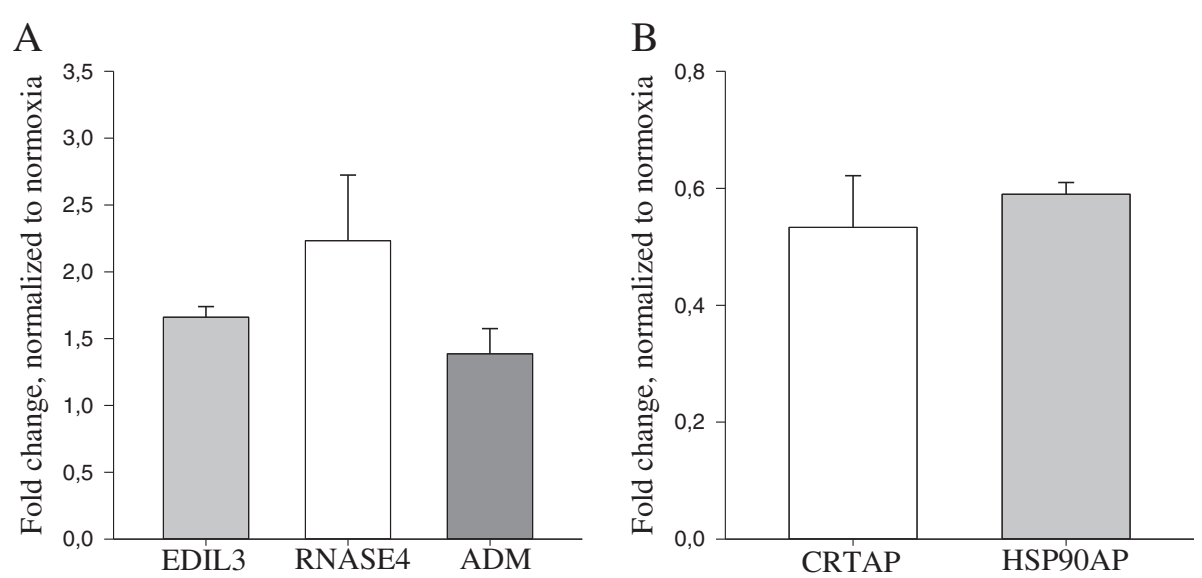

Fig. 2 Hypoxia-induced changes of mRNAs, encoding overrepresented proteins. a mRNAs encoding proteins overrepresented in hypoxic secretomes. b mRNAs encoding proteins overrepresented in normoxic secretomes. mRNA fold changes are expressed using $2^{-\Delta \Delta c t}, n=18$

Most previous proteomic studies have analyzed the content of pooled secretomes rather than of samples obtained from individual donors $[8,23]$. This study indicates that despite similar characteristics, which are routinely assessed for ADSCs, including their differentiation abilities and stromal immunophenotype [19], these cells can differ in the spectrum of produced proteins. Such differences could be explained by the presence of multiple subtypes specialized in the production of particular proteins. Here we demonstrate that analyzed ADSCs cultures differ in the proportion of cells

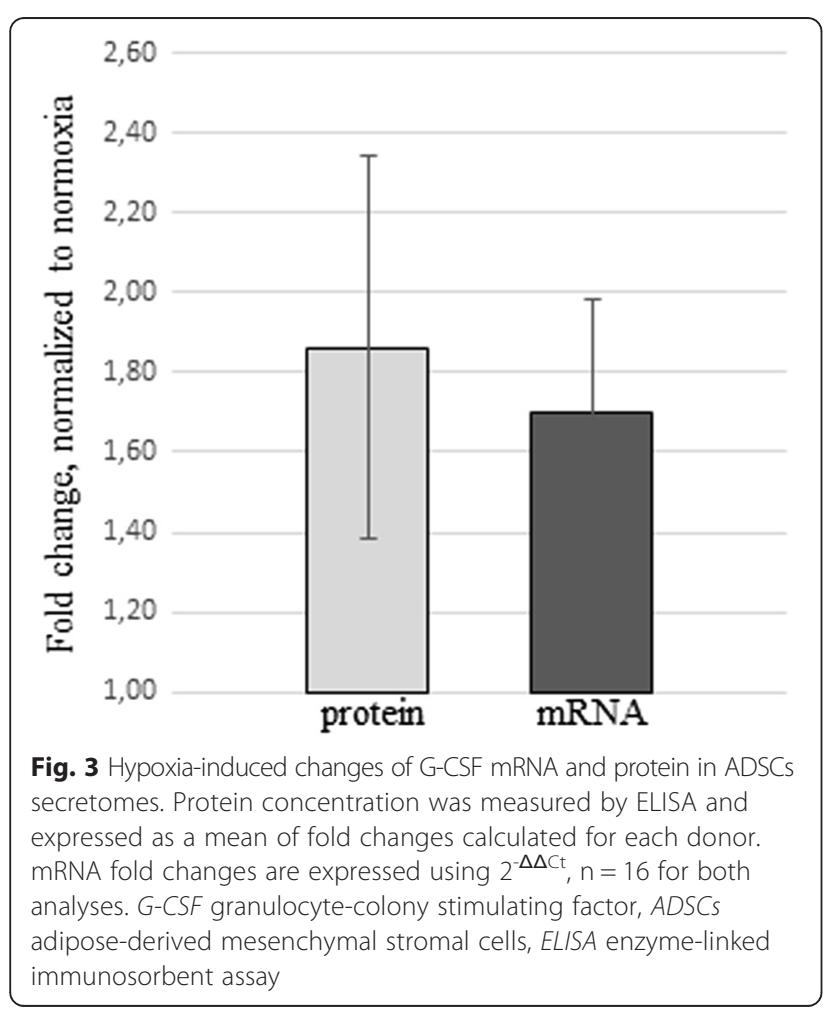

expressing CD146 (MCAM) surface antigen. Furthermore, other membrane proteins, including receptors for hormones and neuromediators could possibly serve as definitive markers for such ADSC subpopulations [28]. Future proteomic analysis of ADSC subpopulations purified according to the expression of those markers is important as it can reveal cells with specialized activities. The usage of such purified cells will allow an increase in the efficiency of ADSC clinical applications and reduce possible side effects.

Consistent with other reports, the largest functional cluster in the ADSC secretome is comprised of ECM proteins. Such protein profile is in line with the stromal characteristics of ADSCs. Adequate production of ECM components is necessary for tissue homeostasis and regeneration [29, 30], because these molecules not only provide a scaffold for cells and soluble molecules, but also regulate angiogenesis and inflammation (i.e., thrombospondin 1 [31], periostin [32], and collagen-derived peptides).

Among proteins common to all ADSC secretomes several molecules not previously associated with ADSCs were identified, including DKK3, olfactomedin-like 3, follistatin-like 1, and prosaposin. These molecules might also contribute to tissue morphogenesis and regeneration induced by ADSCs [33-35]. For example, olfactomedinlike 3 was demonstrated to be important for tumor angiogenesis and vessel stabilization [34]. Considering these data, we can suggest that olfactomedin-like 3 is important for the ability of ADSCs to stimulate the growth of blood vessels. Among variably present molecules, we found several proteins with neurotrophic activities, such as caprin 1, mesencephalic astrocyte-derived neurotrophic factor, meteorin, and neuron derived neurotrophic factor. These factors, together with neurotrophins previously found in ADSCs conditioned medium, including 
BDNF, NGF and GDNF may account for the beneficial action of these cells or their secretomes on the regeneration of brain and peripheral nerves $[4,6]$. Uncovering the content of neurotrophins produced by ADSCs allows the development of a defined preparation of recombinant factors, which might substitute cell therapy in the treatment of neurodegenerative disorders in the future. Such enrichment of ADSCs secretome with neurotrophins is not surprising and is in line with the location of MSCs in close proximity to peripheral nerves in vivo [36].

The in vivo decrease of oxygen tension below its physiological level (2-9 \% $\mathrm{O}_{2}$ for ADSCs) can trigger regeneration processes [37-39]. Cells within a damaged area respond to hypoxia by stabilization of hypoxia inducible factor-1 (HIF-1 $\alpha$ ) [40], which binds to HIF-1 response elements and drives the expression of mRNA and miR encoding genes [41, 42]. In our study we demonstrate that hypoxia stimulates the expression and secretion of angiogenic proteins, including EGF-like EDIL, RNASE4 and adrenomedullin. Ribonuclease 4 was not previously reported as a target of HIF-1a; however, it shares a common promoter with angiogenin, which is regulated by this transcription factor. Previously, angiogenin was found in MSC secretomes [12]. Here we demonstrate that RNASE4 prevails in the ADSCs secretome and might be responsible for angiogenic and anti-apoptotic activities of these cells [43]. This is consistent with known mechanisms of long-term adaptation to hypoxia and supports our previous findings suggesting that hypoxia causes simultaneous up-regulation of angiogenesis stimulators together with down-regulation of its inhibitors in ADSCs $[3,16]$. Prolonged hypoxia also led to the disappearance of proteins regulating osteogenesis and ECM remodeling, such as cartilage-associated protein (uniprot ID\#O75718) and osteoglycin (uniprot ID \#P20774). This is consistent with previous reports, indicating that low $\mathrm{O}_{2}$ concentration suppresses osteogenic differentiation of MSCs [44, 45]. This is also in line with a study of Fraizer TP et al., which nicely quantified the level of proteins in the ADSCs secretomes and demonstrates that exposure of these cells to low $\mathrm{O}_{2}$ reduces ECM components [46].

\section{Conclusions}

Taken together our results suggest that human ADSCs, defined as CD90+/CD73+/CD105+/CD45-/CD31-/PDG FRbeta+/NG2+/CD146 + (-) cells, secrete a large array of proteins, the most represented group being comprised of extracellular matrix components. The number of secreted proteins is largely unaffected by prolonged hypoxia. Variability in the secretion of several proteins from cultured ADSCs of individual subjects suggests that these cells exist as a heterogeneous population containing functionally distinct subtypes, which differ in numbers between donors.
Despite the fact that this requires further investigation, the present study provides a basis for further elucidation of the functional heterogeneity of these cells.

\section{Additional files}

Additional file 1: Table S1. ADSC donor and culture characteristics. Table S2. Common proteins identified in all secretomes of normoxic ADSCs. Table S4. Proteins identified in 1-10 secretomes of normoxic ADSCs. Table S5. List of extracellular matrix proteins found in secretomes of normoxic ADSCs. Table S6. List of proteins involved in blood vessel development, which were found in secretomes of normoxic ADSCs. Table S7. List of proteins involved in neuron differentiation, which were found in secretomes of normoxic ADSCs. (DOCX $118 \mathrm{~kb}$ )

Additional file 2: Figure S1. Representative images of ADSCs undergoing adipogenic or osteogenic differentiation. A1-A7. - Cells incubated in adipogenesis inducing medium for 27 days. C1-C7. - Cells incubated in osteogenesis inducing medium for 21 day. A, B. - Cells stained with Oil-red O to detect lipid accumulations. C, D. - Cells stained with Alizarin red S to detect calcium accumulations. Nuclei were counterstained by hematoxilin. Scale $=100 \mu \mathrm{m}$. (TIFF $1643 \mathrm{~kb}$ )

Additional file 3: Figure S2. Representative flow cytometry charts and plots of additional surface markers expression on ADSC obtained from eight donors (\#2-9). A. - Expression of pericyte markers, PDGFRß and NG2 glycoprotein. Pink areas - specific antibodies, blue areas - isotype antibodies. B. - Expression of CD146 together with CD34 or CD31. (TIFF 262 kb)

Additional file 4: Table S3. Data of transcriptome profiling of normoxic ADSCs. (DOCX 87 kb)

Additional file 5: Figure S3. ADSC response to hypoxia. A. Representative Western blots showing the accumulation of HIF-1alpha after the incubation at $1 \% \mathrm{O}_{2}$ during $48 \mathrm{~h}$. B. - Real time PCR analysis of VEGF mRNA in hypoxic and normoxic ADSCs ( $n=20,{ }^{*}-p<0.05$ vs. normoxia). (TIFF $100 \mathrm{~kb}$ )

\section{Abbreviations}

ADSCs: Adipose-derived mesenchymal stromal cells; BDNF: Brain derived neurotrophic factor; DKK3: Dickkopf-related protein; DTT: Dithiothreitol; ECM: Extracellular matrix; EGF: Epidermal growth factor; G-CSF: Granulocytecolony stimulating factor; GDNF: Glial cell derived neurotrophic factor; HGF: Hepatocyte growth factor; HIF-1: Hypoxia inducible factor-1; IGF-1: Insulinlike growth factor; IGFBP: Insulin-like growth factor-binding protein; IL: Interleukin; LS/MS: Liquid chromatography/mass spectrometry; MCAM: Melanoma cell adhesion molecule; M-CSF: Monocyte colony stimulating factor; MEM: Minimum essential medium; MIF: Macrophage migration inhibitory factor; NG2: Neural/glial antigen 2; NGF: Beta-nerve growth factor; PDGF: Platelet-derived growth factor; PDGFRB: platelet-derived growth factor receptor beta; PEDF: Pigment epitheliumderived factor; RNASE4: Ribonuclease 4; SCF: Stem cell factor; SDF: Stromal cell derived factor; VEGF: Vascular endothelial growth factor.

\section{Competing interests}

The authors declare that they have no competing interests.

\section{Authors' contributions}

NK participated in study design, performed immunophenotyping, and manuscript writing; DK, ML, IB, OP and VL participated in study design, conducted LS/MS profiling, and assisted with manuscript writing; AE assessed HIF-1a activation, performed ELISA and participated in manuscript preparation; LA conducted realtime PCR and assisted with manuscript writing; GS performed FACS analysis, helped with data interpretation, and critically revised manuscript; DI and DA conducted bioinformatic and statistical analysis and were involved in drafting the manuscript; OG cultured ADSC, harvested conditioned medium, and participated in manuscript preparation; VS and KR participated in study design, performed cell culture, differentiation and viability assays, and were involved in revising the manuscript; VG performed study design and manuscript writing. All authors read and approved the final manuscript. 


\section{Acknowledgements}

This study was supported by Russian Science Foundation grant \#14-15-00439 (for proteomic analysis of normoxic and hypoxic secretomes, ADSC differentiation, and immunophenotyping) and \#14-24-00086 (for transcriptome profiling). The authors thank Professor Alexander Bobik (Monash University, Melbourne) for helpful discussion of the data included in the manuscript.

\section{Author details}

${ }^{1}$ Faculty of Medicine, Lomonosov Moscow State University, 31-5, Lomonosovsky av, Moscow 119191, Russia. ${ }^{2}$ Department of Molecular Biology and Genetics, SRI of Physical-Chemical Medicine, 1a, Malaya Pirogovskaya, Moscow 119435, Russia.

Received: 13 February 2015 Revised: 18 February 2015

Accepted: 20 October 2015

Published online: 11 November 2015

\section{References}

1. Zuk PA, Zhu M, Mizuno H, Huang J, Futrell JW, Katz AJ, et al. Multilineage cells from human adipose tissue: implications for cell-based therapies. Tissue Eng. 2001;7:211-28.

2. Rehman J, Traktuev D, Li J, Merfeld-Clauss S, Temm-Grove CJ, Bovenkerk JE et al. Secretion of angiogenic and antiapoptotic factors by human adipose stromal cells. Circulation. 2004;109:1292-8.

3. Rubina K, Kalinina N, Efimenko A, Lopatina T, Melikhova V, Tsokolaeva Z, et al. Adipose stromal cells stimulate angiogenesis via promoting progenitor cell differentiation, secretion of angiogenic factors, and enhancing vessel maturation. Tissue Eng Part A. 2009;15:2039-50.

4. Lopatina T, Kalinina N, Karagyaur M, Stambolsky D, Rubina K, Revischin A, et al. Adipose-derived stem cells stimulate regeneration of peripheral nerves: BDNF secreted by these cells promotes nerve healing and axon growth de novo. PLoS One. 2011;6:e17899.

5. Lee SM, Lee SC, Kim SJ. Contribution of human adipose tissue-derived stem cells and the secretome to the skin allograft survival in mice. J Surg Res. 2014:188:280-9.

6. Wright KT, Uchida K, Bara JJ, Roberts S, El Masri W, Johnson WE. Spinal motor neurite outgrowth over glial scar inhibitors is enhanced by coculture with bone marrow stromal cells. Spine J. 2014;14:1722-33.

7. Lopatina T, Bruno S, Tetta C, Kalinina N, Porta M, Camussi G. Platelet-derived growth factor regulates the secretion of extracellular vesicles by adipose mesenchymal stem cells and enhances their angiogenic potential. Cell Commun Signal. 2014;12:26.

8. Kupcova SH. Proteomic techniques for characterisation of mesenchymal stem cell secretome. Biochimie. 2013;95:2196-211.

9. Wei X, Du Z, Zhao L, Feng D, Wei G, He Y, et al. IFATS collection: the conditioned media of adipose stromal cells protect against hypoxia-ischemia-induced brain damage in neonatal rats. Stem Cells. 2009;27:478-88.

10. Chang CP, Chio CC, Cheong CU, Chao CM, Cheng BC, Lin MT. Hypoxic preconditioning enhances the therapeutic potential of the secretome from cultured human mesenchymal stem cells in experimental traumatic brain injury. Clin Sci (Lond). 2013;124:165-76.

11. Dzhoyashvili NA, Efimenko A, Kochegura TN, Kalinina NI, Koptelova NV, Sukhareva $\mathrm{O}$, et al. Disturbed angiogenic activity of adipose-derived stromal cells obtained from patients with coronary artery disease and diabetes mellitus type 2. J Transl Med. 2014;12(1):337. doi:10.1186/s1296 7-014-0337-4.

12. Efimenko A, Dzhoyashvili N, Kalinina N, Kochegura T, Akchurin R, Tkachuk V, et al. Adipose-derived mesenchymal stromal cells from aged patients with coronary artery disease keep mesenchymal stromal cell properties but exhibit characteristics of aging and have impaired angiogenic potential. Stem Cells Transl Med. 2014:3:32-41. doi:10.5966/sctm.2013-0014.

13. Choudhery MS, Badowski M, Muise A, Pierce J, Harris DT. Donor age negatively impacts adipose tissue-derived mesenchymal stem cell expansion and differentiation. J Transl Med. 2014;12:8. doi:10.1186/1479-5876-12-8.

14. Gremmels H, Teraa M, Quax PH, den Ouden K, Fledderus JO, Verhaar MC Neovascularization capacity of mesenchymal stromal cells from critical limb ischemia patients is equivalent to healthy controls. Mol Ther. 2014;22:1960-70. doi:10.1038/mt.2014.161.

15. Potier E, Ferreira E, Andriamanalijaona R, Pujol JP, Oudina K, Logeart-Avramoglou $D$, et al. Hypoxia affects mesenchymal stromal cell osteogenic differentiation and angiogenic factor expression. Bone. 2007;40:1078-87.
16. Efimenko A, Starostina E, Kalinina N, Stolzing A. Angiogenic properties of aged adipose derived mesenchymal stem cells after hypoxic conditioning. J Transl Med. 2011;9:10.

17. Wang J, Ding F, Gu Y, Liu J, Gu X. Bone marrow mesenchymal stem cells promote cell proliferation and neurotrophic function of Schwann cells in vitro and in vivo. Brain Res. 2009;1262:7-15.

18. Ohnishi S, Yasuda T, Kitamura S, Nagaya N. Effect of hypoxia on gene expression of bone marrow-derived mesenchymal stem cells and mononuclear cells. Stem Cells. 2007;25:1166-77.

19. Dominici M, Le Blanc K, Mueller I, Slaper-Cortenbach I, Marini F, Krause D, et al. Minimal criteria for defining multipotent mesenchymal stromal cells. The International Society for Cellular Therapy position statement. Cytotherapy. 2006:8:315-7.

20. Laemmli UK. Cleavage of structural proteins during the assembly of the head of bacteriophage T4. Nature. 1970;227:680-5.

21. Peferoen M. Blotting with plate electrodes. Methods Mol Biol. 1988;3:395-402 doi:10.1385/0-89603-126-8:395

22. Huang DW, Sherman BT, Lempicki RA. Systematic and integrative analysis of large gene lists using DAVID Bioinformatics Resources. Nat Protoc. 2009;4(1):44-57.

23. Kapur SK, Katz AJ. Review of the adipose derived stem cell secretome. Biochimie. 2013;95(12):2222-8. doi:10.1016/j.biochi.2013.06.001.

24. Lee MJ, Kim J, Kim MY, Bae YS, Ryu SH, Lee TG, et al. Proteomic analysis of tumor necrosis factor-a-induced secretome of human adipose tissuederived mesenchymal stem cells. J Proteome Res. 2010;9(4):1754-62. doi:10.1021/pr900898n

25. Rabouille C, Malhotra V, Nickel W. Diversity in unconventional protein secretion. J Cell Sci. 2012:125(Pt 22):5251-5. doi:10.1242/jcs.103630.

26. Deora AB, Kreitzer G, Jacovina AT, Hajjar KA. An annexin 2 phosphorylation switch mediates p11-dependent translocation of annexin 2 to the cell surface. J Biol Chem. 2004;279:43411-8. doi:10.1074/jbc.M408078200

27. Tveit H, Akslen LK, Fagereng GL, Tranulis MA, Prydz K. A secretory Golgi bypass route to the apical surface domain of epithelial MDCK cells. Traffic. 2009;10:1685-95. doi:10.1111/j.1600-0854.2009.00984.x.

28. Kotova PD, Sysoeva VY, Rogachevskaja OA, Bystrova MF, Kolesnikova AS, Tyurin-Kuzmin PA, et al. Functional expression of adrenoreceptors in mesenchymal stromal cells derived from the human adipose tissue. Biochim Biophys Acta. 2014;1843(9):1899-908. doi:10.1016/j.bbamcr. 2014.05.002.

29. Pickup MW, Mouw JK, Weaver VM. The extracellular matrix modulates the hallmarks of cancer. EMBO Rep. 2014;15(12):1243-53. doi:10.15252/embr. 201439246.

30. Mammoto T, Ingber DE. Mechanical control of tissue and organ development. Development. 2010;137(9):1407-20. doi:10.1242/dev.024166.

31. Stenina-Adognravi O. Invoking the power of thrombospondins: regulation of thrombospondins expression. Matrix Biol. 2014;37C:69-82.

32. Liu AY, Zheng H, Ouyang G. Periostin, a multifunctional matricellular protein in inflammatory and tumor microenvironments. Matrix Biol. 2014;37C:150-6.

33. Aoka Y, Johnson FL, Penta K, Hirata Ki K, Hidai C, Schatzman R, et al. The embryonic angiogenic factor Del1 accelerates tumor growth by enhancing vascular formation. Microvasc Res. 2002;64:148-61.

34. Miljkovic-Licina M, Hammel P, Garrido-Urbani S, Lee BP, Meguenani M, Chaabane C, et al. Targeting olfactomedin-like 3 inhibits tumor growth by impairing angiogenesis and pericyte coverage. Mol Cancer Ther. 2012:11:2588-99.

35. Chaly Y, Hostager B, Smith S, Hirsch R. Follistatin-like protein 1 and its role in inflammation and inflammatory diseases. Immunol Res. 2014;59(1-3):266-72. doi:10.1007/s12026-014-8526-z.

36. Méndez-Ferrer S, Michurina TV, Ferraro F, Mazloom AR, MacArthur BD, Lira SA, et al. Mesenchymal and haematopoietic stem cells form a unique bone marrow niche. Nature. 2010;466:829-34.

37. Imanirad P, Dzierzak E. Hypoxia and HIFs in regulating the development of the hematopoietic system. Blood Cells Mol Dis. 2013;51:256-63.

38. Sanada F, Kim J, Czarna A, Chan NY, Signore S, Ogorek B, et al. c-Kit-positive cardiac stem cells nested in hypoxic niches are activated by stem cell factor reversing the aging myopathy. Circ Res. 2014;114:41-55.

39. Zhang K, Zhao T, Huang X, Wu LY, Wu K, Zhu LL, et al. Notch1 mediates postnatal neurogenesis in hippocampus enhanced by intermittent hypoxia. Neurobiol Dis. 2014;64:66-78.

40. Palazon A, Goldrath AW, Nizet V, Johnson RS. HIF transcription factors, inflammation, and immunity. Immunity. 2014;41:518-28. 
41. Jacobs LA, Bewicke-Copley F, Poolman MG, Pink RC, Mulcahy LA, Baker I, et al. Meta-analysis using a novel database, miRStress, reveals miRNAs that are frequently associated with the radiation and hypoxia stress-responses. PLoS One. 2013:8:e80844

42. Nie Y, Han BM, Liu XB, Yang JJ, Wang F, Cong XF, et al. Identification of microRNAs involved in hypoxia- and serum deprivation-induced apoptosis in mesenchymal stem cells. Int J Biol Sci. 2011;7:762-8.

43. Li S, Sheng J, Hu JK, Yu W, Kishikawa H, Hu MG, et al. Ribonuclease 4 protects neuron degeneration by promoting angiogenesis, neurogenesis, and neuronal survival under stress. Angiogenesis. 2013;16:387-404

44. Xu N, Liu H, Qu F, Fan J, Mao K, Yin Y, et al. Hypoxia inhibits the differentiation of mesenchymal stem cells into osteoblasts by activation of Notch signaling. Exp Mol Pathol. 2013;94:33-9.

45. Benjamin S, Sheyn D, Ben-David S, Oh A, Kallai I, Li N, et al. Oxygenated environment enhances both stem cell survival and osteogenic differentiation. Tissue Eng Part A. 2013;19:748-58.

46. Frazier TP, Gimble JM, Kheterpal I, Rowan BG. Impact of low oxygen on the secretome of human adipose-derived stromal/stem cell primary cultures. Biochimie. 2013;95(12):2286-96. doi:10.1016/j.biochi.2013.07.011.

47. Huang DW, Sherman BT, Lempicki RA. Bioinformatics enrichment tools: paths toward the comprehensive functional analysis is of large gene lists. Nucleic Acids Res. 2009;37(1):1-13.

\section{Submit your next manuscript to BioMed Central and take full advantage of:}

- Convenient online submission

- Thorough peer review

- No space constraints or color figure charges

- Immediate publication on acceptance

- Inclusion in PubMed, CAS, Scopus and Google Scholar

- Research which is freely available for redistribution 